\title{
EAl Endorsed Transactions

\section{Comparative Analysis of Face Detection Using Linear Binary Techniques and Neural Network Approaches}

\author{
Laraib Mughal $^{1, *}$, Kaneez Laila Bhatti ${ }^{2}$, Faheem Khuhawar $^{2}$ and Fawwad Hassan Jaskani ${ }^{3}$ \\ ${ }^{1}$ Author, Mehran University of Engineering and Technology, Jamshoro \\ ${ }^{2}$ Co-Author, Mehran University of Engineering and Technology, Jamshoro \\ ${ }^{3}$ Co-Author, Khawaja Fareed University of Engineering and Information Technology, Rahim Yar Khan
}

\section{Abstract}

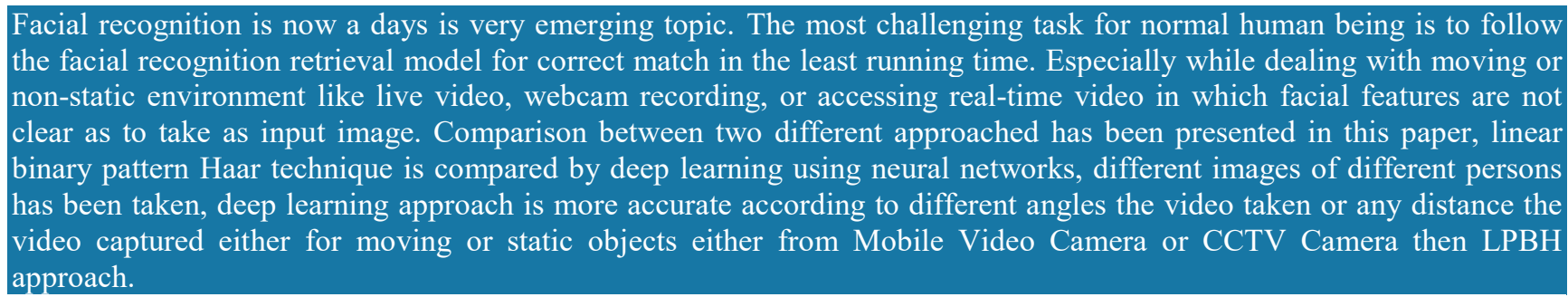

Keywords: Facial recognition, CNN, LBP, Neural Networks, HAAR Classifier.

Received on 26 August 2018, accepted on 23 October 2018, published on 29 October 2018

Copyright (C) 2018 Laraib Mughal et al., licensed to EAI. This is an open access article distributed under the terms of the Creative Commons Attribution licence (http://creativecommons.org/licenses/by/3.0/), which permits unlimited use, distribution and reproduction in any medium so long as the original work is properly cited.

doi: 10.4108/eai.18-10-2018.159712

"Corresponding author. Email:Laraibmugha127@gmail.com

\section{Introduction}

For Face Detection we are using the HAAR Image Processing Classifier for detecting whether any face is present if yes then how many and returns array of faces but since this algorithm is not accurate in detecting faces as it doesn't work mostly on slightly angled faces which is another reason that we have concluded Deep Learning the most accurate but since HAAR doesn't require high computing so it is very quick and almost real-time so it is suitable for real-time detection in systems where performance is bottleneck.

So first we provided training images and loop over them one by one, after finding any face we will directly put that face location in an array variable here not that no any facial matching points are extracted just complete face location area is stored in numerical array form in one array variable one by one. After training now it is time to test, so we will take new images of that person and for detection it is required that only single face should be present since this techniques lacks for multiple detection at a time.
It will be then initialize LBP Recognizer and pass it on the array of that Faces we have stored and we pass our Face location of detected face in test image from HAAR. Now what it does that it will match the histogramatic changes or patterns in both images if there exist any similarity between the both pattern then it consider a match otherwise it will return some name but with very low confidence, in this we cannot determine directly whether face is unknown or not so we have added a condition in our code that if the confidence is less than $60 \%$ then we consider that result as unknown by ignoring the Label or name returned by algorithm, Simulating the result conclude that the deep learning approach is more accurate according to different angles the video taken or any distance the video captured either for moving or static objects either from Mobile Video Camera or CCTV Camera then LPBH approach. 


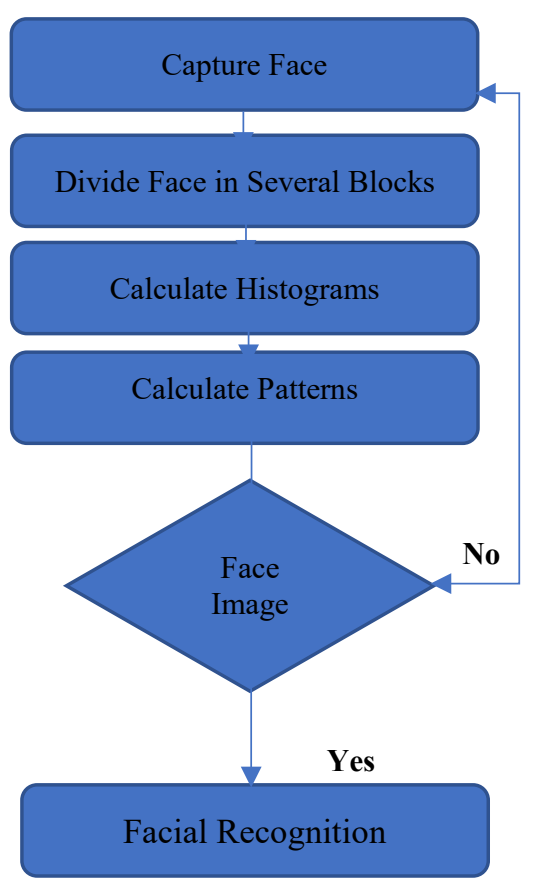

Figure 1. Basic Terminology

\section{Literature Review}

Human face detection is one of the intriguing classes with regards to biometric learning which has pulled in much consideration as of late. There are different methodologies which have been produced for this reason. Thinking about opposite sides of a coin, every strategy has its solid and frail focuses. The six principle techniques are information - based strategies, appearance-based techniques, include invariant strategies, geometry-based techniques, format based strategies, and model-based techniques [1] The reason for this short audit paper [2] is to introduce, order and assess some new face detection techniques utilizing four ordinary learning machine. The execution and the other assessment parameters of these strategies contrast with one another all together with acquaint critical techniques and furthermore with state favourable circumstances and inconveniences of related works. [2] The focal point of this paper [3] is to construct a safe confirmation framework with face, area and motion acknowledgment as segments. Client motions and area information are a succession of time arrangement; in this way, in this paper we propose to utilize unsupervised learning in the long transient memory repetitive neural system to effectively figure out how to perceive, gathering and segregate client signals and area. In addition, a bunching based strategy is additionally actualized for perceiving signals and area. [1][3]

\subsection{Linear Binary Pattern}

The LBP administrator can be viewed as a really bringing together way to deal with the customarily unique factual and basic models of surface investigation. Surface is depicted as far as small scale natives and their measurable situation rules. Alternatively, the natives might be combined with a correlative proportion of nearby picture differentiate, which estimates the quality of the natives. [4]

Because of the very idea of the issue, PC researchers, neuroscientists and clinicians all offer a distinct fascination for this field. In plain words, it is a PC application for consequently distinguishing an individual from a still picture or video outline. One of the approaches to achieve this is by looking at chosen highlights from the picture and a facial database. There are hundreds if not thousand components related with this. In this paper the absolute most normal techniques accessible including uses of neural system in facial acknowledgment are contemplated and contrasted with deference with their execution. [5]

Techniques, for example, Principal Component investigation (PCA), Independent part examination (ICA), Linear Discriminant Analysis (LDA), have been the dominating ones to be utilized. In any case, with upgrades required in the past methodologies Neural Networks based acknowledgment resembled aid to the business. It improved the acknowledgment as well as the proficiency of the procedure. Picking Backpropagation as the learning strategy was unmistakably out of its productivity to perceive nonlinear faces with an acknowledgment proportion of over $90 \%$ and execution time of just couple of moments.[6] Novel methodology is introduced to face recognition which considers both shape and surface data to speak to the face. The face region is first separated into little areas from which Local Binary Pattern (LBP) histograms are extricated and linked into a solitary, spatially improved element histogram productively speaking to the face picture. Broad test look into demonstrates the predominance of the proposed strategy in regard of its straightforwardness and effectiveness in extremely quick component extraction. [7]

\subsection{Principle Component Analysis}

There has been an inclination in the $\mathrm{PC}$ vision network to lean toward LDA over PCA. This is essentially in light of the fact that LDA manages segregation between classes while PCA does not focus on the hidden class structure. At the point when the preparation set is little, PCA can outflank LDA. At the point when the quantity of tests is vast and delegate for each class, LDA outflanks PCA. Numerous works broke down the contrasts between these two techniques, however no work examined the likelihood of intertwining them. As we would see it, the clear solid relationship of LDA and PCA, particularly when frontal perspectives are utilized and PCA is connected before LDA, debilitated the combination of such calculations. Notwithstanding, it ought to be noticed that LDA and PCA are not as associated as one can might suspect, as the LDA change connected to the vital segments can create a component space altogether unique in relation to the PCA one. Consequently, the combination of LDA and PCA for face recognition and check is worth of hypothetical and exploratory examination. [8] An effective face recognition approach depends intensely on the specific decision of the highlights utilized by the pattern classifier Neural based Face recognition is powerful and has preferable execution of increasingly over $90 \%$ acknowledgment proportion [9]

Nearby Binary Pattern (LBP) and its changed models Multivariate Local Binary Pattern (MLBP), Centre Symmetric Local Binary Pattern (CS-LBP) and Local Binary Pattern 
Variance (LBPV) are researched. Facial highlights are removed and thought about utilizing $\mathrm{K}$ closest neighbour order calculation. G-insights remove measure is utilized for characterization. Examinations were directed on JAFFE female, CMU-PIE and FRGC version2 databases. The outcomes demonstrates that CS-LBP reliably [9], [10]

In this paper, another methodology EMB-LTP for face recognition is introduced. Serious examinations are finished. Examinations are made among Basic LBP, Basic LTP, Basic MB-LBP, MB-LTP, ELBP, ELTP, and EMB-LBP as methodologies for face recognition. [10]

The trial results on the Yale face database demonstrate that the proposed administrator EMB-LTP is similar with LTP and ELTP yet is better than the other tried methodologies. Since the Yale face database is little and basic, the prevalence of the proposed EMB-LTP remain not affirmed. In this way, we intend to do more analyses on enormous and complex face databases. [11]

\subsection{Linear Discriminant Regression Classification}

(LDRC) is a successful technique created in the ongoing years on point of giving improvement to the exactness of Face Recognition (FR) based frameworks. [12] There are different methodologies which have been produced for this reason. Thinking about opposite sides of a coin, every strategy has its solid and frail focuses. The six principle techniques are information - based strategies, appearance-based techniques, include invariant strategies, geometry-based techniques, format based strategies, and model-based techniques [1] The reason for this short audit paper [2] is to introduce, order and assess some new face detection techniques utilizing four ordinary learning machine. The execution and the other assessment parameters of these strategies contrast with one another all together with acquaint critical techniques and furthermore with state favourable circumstances and inconveniences of related works. [2] The focal point of this paper [3] is to construct a safe confirmation framework with face, area and motion acknowledgment as segments. Client motions and area information are a succession of time arrangement; in this way, in this paper we propose to utilize unsupervised learning in the long transient memory repetitive neural system to effectively figure out how to perceive, gathering and segregate client signals and area. In addition, a bunching based strategy is additionally actualized for perceiving signals and area.[13]

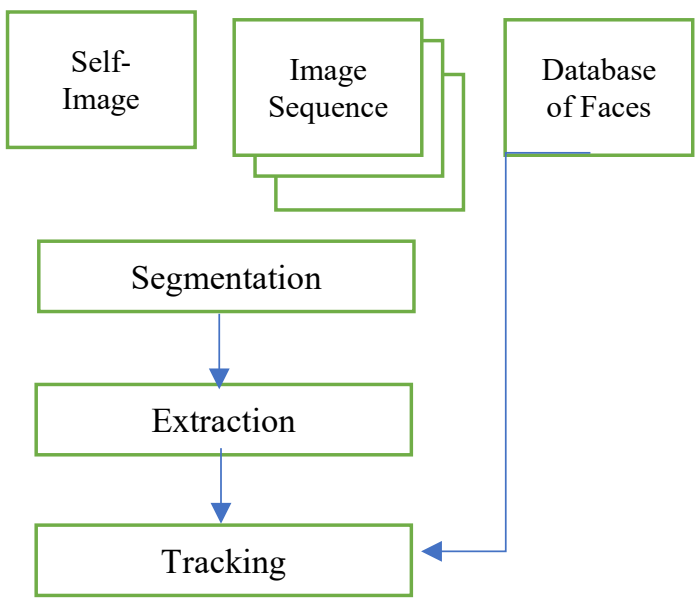

Figure 2. Identification of One's Persons Face

\section{Deep learning techniques}

When contrasted with customary machine learning approaches, deep learning based techniques have demonstrated better exhibitions as far as exactness and speed of handling in picture recognition. This paper proposes an adjusted Convolutional Neural Network (CNN) design by adding two standardization tasks to two of the layers. The standardization activity which is group standardization given speeding up of the system. CNN design was utilized to separate particular face features and Softmax classifier was utilized to group faces in the completely associated layer of CNN. In the trial part, Georgia Tech Database demonstrated that the proposed methodology has improved the face recognition execution with better recognition results. [14]

To handle with these conditions and to accomplish the motivation behind face detection or recognition, it is important to make the near investigation of different techniques. There are different techniques which have been created and utilized. Every system has its very own qualities, favourable circumstances, weaknesses, execution, delegate work and so on. In this paper, we have present the relative investigation of different techniques, for example, face recognition utilizing PCA,DCT change, LDA, neural networks, and so forth. Different parameters, including benefits and faults of the considerable number of techniques are considered to choose which system is increasingly valuable in future. [15]

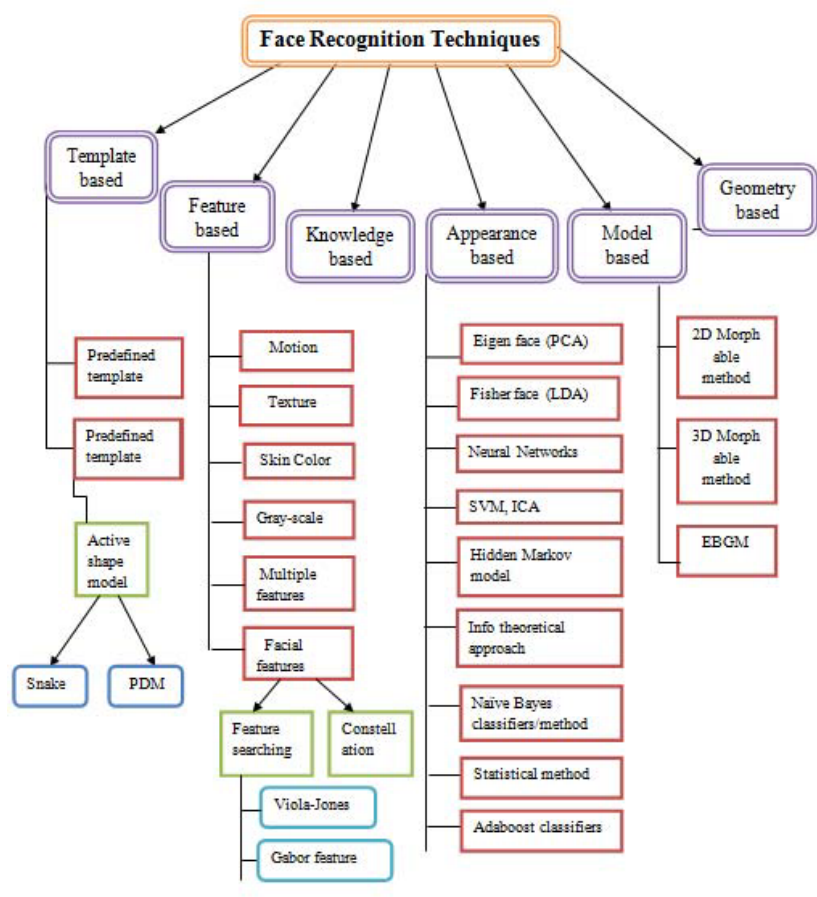

Figure 3. Face Recognition Techniques [3]

With the deep learning in various territories of accomplishment, past different strategies, set off another influx of neural system improvement. The idea of deep learning began from the counterfeit neural system, generally, alludes to a class of neural networks with deep structure of the compelling 
preparing strategies. As an incredible innovation to acknowledge man-made brainpower, deep learning has been generally utilized in penmanship computerized recognition, measurement rearrangements, discourse recognition, picture appreciation, machine interpretation, protein structure expectation and feeling recognition. In this paper, we centre around the examination hotspots of face recognition dependent on profundity learning in the field of biometrics, Combined with the pertinent hypothesis and techniques for profundity learning, face recognition innovation, along the Order of profundity learning, in view of the profundity of learning face recognition, face recognition application to begin look into [16][17]

This undertaking presents face recognition dependent on Difference of Gaussian and highlight extraction utilizing Discriminative Robust Local Binary pattern (DRLBP) approach. The Median channel is utilized to extricate the half and half features and the pyramids are created after the face granulation. At that point, DoG pyramid will be shaped from progressive emphases of Gaussian pictures. By this granulation, facial features are isolated at various goals to give edge data, commotion, smoothness and haziness present in a face picture. In highlight extraction arrange, this binary face format act like a veil to extricate nearby surface data utilizing Discriminative Robust Local binary pattern. This strategy is effective to face recognition since it is less delicate to light and scaling. . It diminishes the computational time multifaceted nature and space unpredictability. This proposed methodology lessens the calculation time and furthermore builds the productivity [18]

The proposed strategy takes a shot at 3D polygonal lattices we right off the bat need to get genuine faces and to speak to them as polygonal surfaces. The Ambient Intelligence setting, in which we are executing face recognition, requires quick client enlistment to abstain from irritating holding up time. Generally, most 3D face recognition strategies take a shot at a range picture of the face, caught with laser or organized light scanner. This sort of gadgets offer high goals in the caught information, however they are unreasonably moderate for a continuous face securing. Face undesirable movement amid catching could be another issue, while laser checking couldn't be innocuous to the eyes. [19], [20]

Identified faces are passed to a socioeconomics classifier which utilizes a similar engineering as the face locator. This statistic classifier is incredibly quick, and conveys blunder rates somewhat superior to the best known classifiers. To counter the unconstrained and loud detecting condition, statistic data is coordinated crosswise over time for every person. In this way, the last statistic grouping joins gauges from numerous facial detections so as to decrease blunder rate. The whole framework forms 10 outlines for each second on an $800 \mathrm{MHz}$ Intel PIII. [21], [22]

The vast majority of existing face detection calculations consider a face detection as binary (two-class) grouping issue. Despite the fact that it looks a straightforward arrangement issue, it is perplexing to assemble a decent face classifier. In this manner, learning-based methodologies, for example, neural system based strategies or supports vector machine (SVM) techniques, have been proposed to locate a decent classifiers [2][3][4][5]. The greater part of proposed calculations use pixel esteems as features. Nonetheless, they are delicate to light conditions and clamors [6]. Papageorgiou et al. [7] utilized new component, it is called Haar-like features. These features encode contrasts in normal powers between two rectangular districts, and they can extricate surface without relying upon outright forces Local Binary Pattern (LBP) features have performed great in different applications, including surface characterization and division, picture recovery and surface examination. The first
LBP administrator marks the pixels of a picture by thresholding the 3-by-3 neighborhood of every pixel with the inside pixel esteem and thinking about the outcome as a binary number. Figure 3 demonstrates a case of LBP computation.[23]-[25]

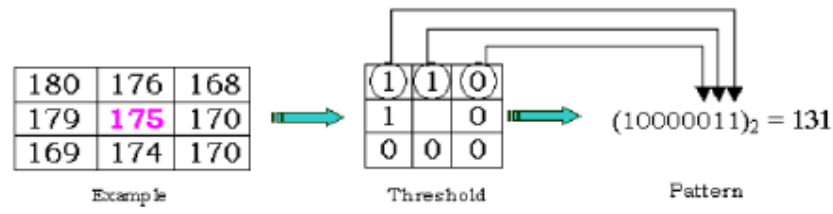

In this paper some of the most common techniques available including applications of neural network in facial recognition are studied and compared with respect to their performance with LBP

Face image is isolated into a few districts and LBP is connected and features are extricated over the area. These features are concatenated to form face descriptor. Although face recognition with local binary pattern has been demonstrated to be a vigorous calculation, it experiences overwhelming computational burden because of the high dimensional element vectors that are extricated by linking the LBP histograms from every local area. Another multichannel filter based Gabor wavelet is planned dependent on hypothesis and common sense. Its middle recurrence is the range from low recurrence to high recurrence, its direction is 6 and scale is 6 .

It can remove the element of low quality outward appearance picture target, and have well powerful for automatic outward appearance recognition

The deep learning calculation is utilized in this framework to dispense with the mistakes caused in this framework and furthermore to further advance the exhibition of the framework. Deep Learning is tied in with learning various dimensions of portrayal and deliberation that help to bode well or data of data, for example, pictures, sound, and content and so on. The info picture or data is found out and the data is dissected to deliver the scholarly yield data.

\section{Methodology}

Pictures of different persons has been captured by hyperspectral cameras using CCTV and Mobile phones, we have compared these images by implementing Deep learning approach and LBPH on them separately. Created the images dataset. And detect faces by histograms and after detecting we extract the location of face on images. After that we have applied two algorithms on these datasets separately. Compare these results and concluded this. As shown in figure below. 


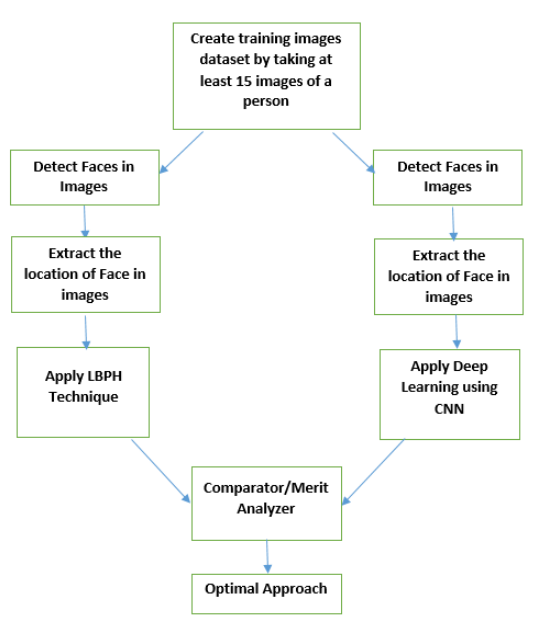

Figure 4. Proposed Methodology

So first we provided training images and loop over them one by one, after finding any face we will directly put that face location in an array variable here not that no any facial matching points are extracted just complete face location area is stored in numerical array form in one array variable one by one. After training now it is time to test, so we will take new images of that person and for detection it is required that only single face should be present since this techniques lacks for multiple detection at a time.

It will be then initialize LBP Recognizer and pass it on the array of that Faces we have stored and we pass our Face location of detected face in test image from HAAR. Now what it does that it will match the histogramatic changes or patterns in both images if there exist any similarity between the both pattern then it consider a match otherwise it will return some name but with very low confidence, in this we cannot determine directly whether face is unknown or not so we have added a condition in our code that if the confidence is less than $60 \%$ then we consider that result as unknown by ignoring the Label or name returned by algorithm

\section{Results}

Mobile video and CCTV video has been captured on the same time and we have applied LBPH and Deep learning approaches in such a way to compare the accuracy. Video has been captured by different angles.

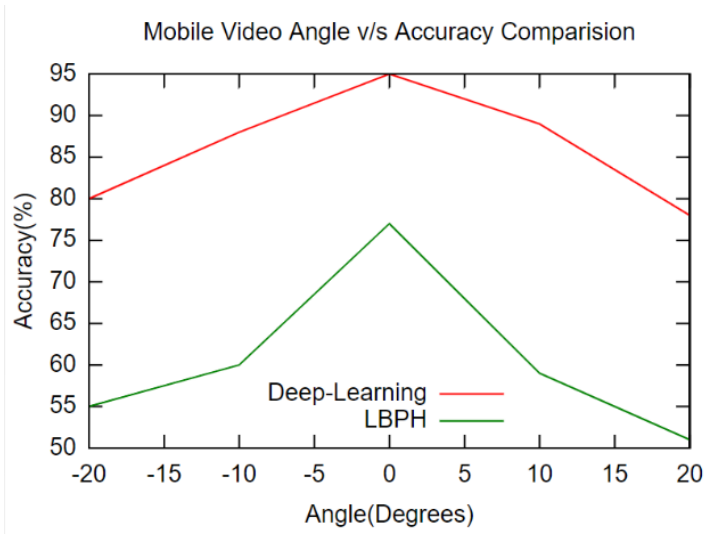

Figure 5. Comparison of LBPH and Deep Learning approach for Mobile Video at different angles

Deep learning has been shown a better approach then haar classifier by comparing different angles of video, at angle zero the accuracy of haar classifier is $75 \%$ while deep learning approach has $95 \%$ accuracy as shown in figure 4 .

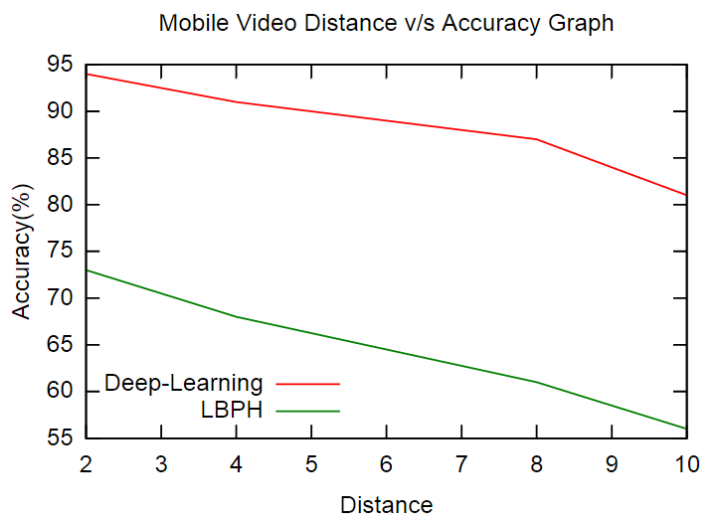

Figure 6. Comparison of Mobile Video Distance with Accuracy Graphs

At different distance video has been taken and from very far distance i.e. $10 \mathrm{~km}$ the accuracy of deep learning approach is $82 \%$ greater than LPBH 


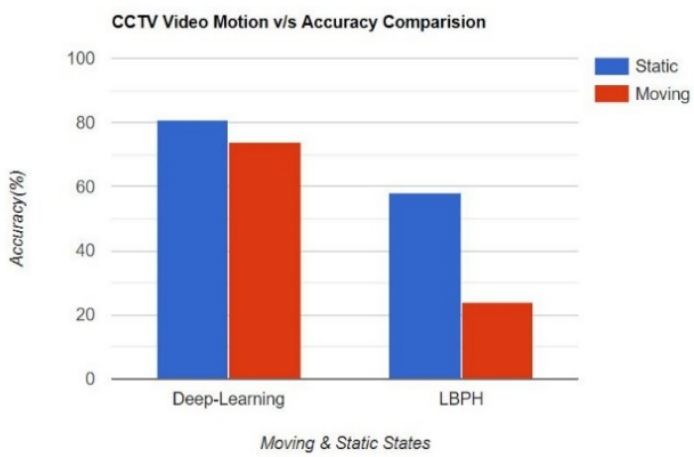

Figure 7. Histogram shows the CCTV video Motion comparison of both algorithms i.e. LPBH and Deep Learning

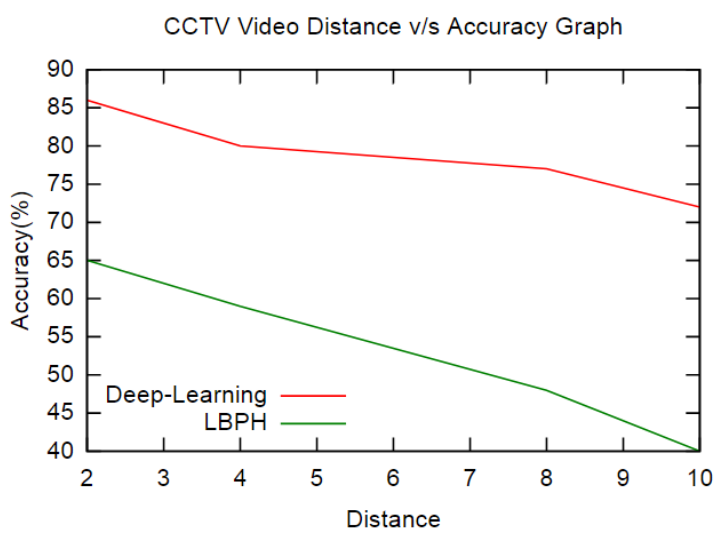

Figure 8. CCTV video Distance Comparison

At different distance CCTV video has been taken and from very far distance i.e. $10 \mathrm{~km}$ the accuracy of deep learning approach is $22 \%$ greater than LPBH CCTV Video Angle v/s Accuracy Comparision

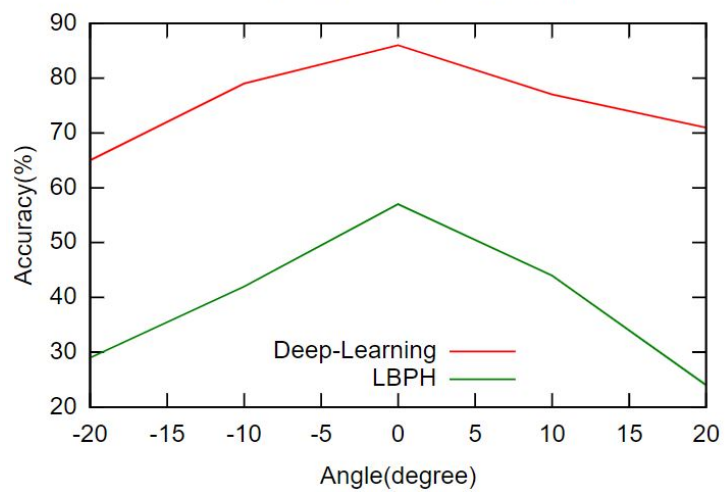

Figure 9. Accuracy according to Different angles captured by CCTV

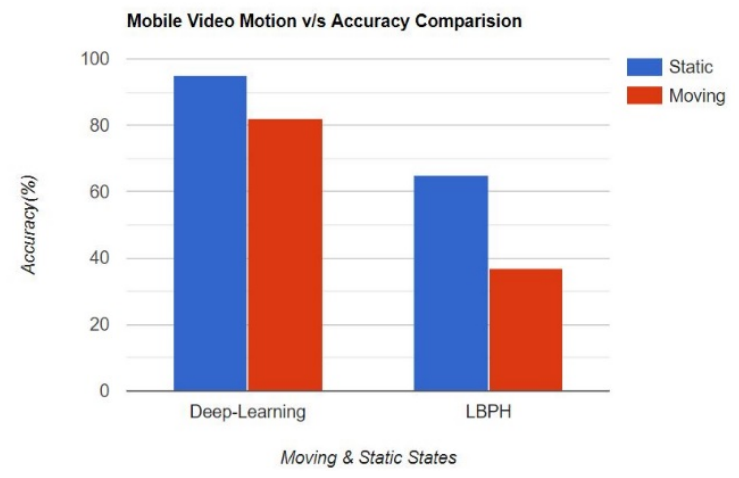

Figure 10. Static and Moving Objects accuracy in Mobile Video Camera

\section{Conclusion}

Simulating the result conclude that the deep learning approach is more accurate according to different angles the video taken or any distance the video captured either for moving or static objects either from Mobile Video Camera or CCTV Camera then LPBH approach.

\section{References}

[1] F. Navabifar, M. Emadi, R. Yusof, and M. Khalid, "A short review paper on Face detection using Machine learning," Proc. Int. Conf. Image Process. Comput. Vision, Pattern Recognit., no. 1, p. 1, 2011.

[2] B. Rexha, G. Shala, and V. Xhafa, "Increasing trust worthiness of face authentication in mobile devices by modeling gesture behavior and location using neural networks," Futur. Internet, vol. 10, no. 2, pp. 1-17, 2018.

[3] Gupta, "Face Detection and Recognition using Local," no. December, pp. 7923-7929, 2016.

[4] M. U. Rahman, "A comparative study on face recognition techniques and neural network," 2012.

[5] S. Tikoo and N. Malik, "Detection, Segmentation and Recognition of Face and its Features Using Neural Network," 2017.

[6] Z. Wen and W. Wenwei, "Face recognition based on local binary pattern and deep learning," vol. 35 , no. 1 , pp. 989-994, 2015.

[7] S. Kumar and H. Kaur, "Face RecognitionTechniques: Classification And Comparisons," Int. J. Inf. Technol. Knowl. Manag., vol. 5, no. 2, pp. 361-363, 2012.

[8] K. Meena and A. Suruliandi, "Local binary patterns and its variants for face recognition," Int. Conf. Recent Trends Inf. Technol. ICRTIT 2011, pp. 782-786, 2011.

[9] C. Taouche, M. C. Batouche, M. Chemachema, A. Taleb-Ahmed, and M. Berkane, "New face recognition method based on local binary pattern histogram," STA 2014 - 15th Int. Conf. Sci. Tech. Autom. Control Comput. Eng., pp. 508-513, 2014. 
[10] K. Shailaja and B. Anuradha, "Effective face recognition using deep learning based linear discriminant classification," 2016 IEEE Int. Conf. Comput. Intell. Comput. Res. ICCIC 2016, pp. 1-6, 2017.

[11] K. Dang and S. Sharma, "Review and comparison of face detection algorithms," Proc. 7th Int. Conf. Conflu. 2017 Cloud Comput. Data Sci. Eng., pp. 629-633, 2017.

[12] T. Ensari and M. Gunay, "Comparison of face recognition algorithms," 2017 25th Signal Process. Commun. Appl. Conf., pp. 1-4, 2017.

[13] U. Aiman, V. P. Vishwakarma, and C. Technology, "Face Recognition Using Modified," Iccnt, pp. 3-7, 2017.

[14] L. Xu, M. Fei, W. Zhou, and A. Yang, "Face expression recognition based on convolutional neural network," ANZCC 2018 - 2018 Aust. New Zeal. Control Conf., pp. 115-118, 2019.

[15] M. Sharma and D. Verma, "A Comparative Analysis on Various Techniques of Fingerprint Recognition," vol. 2, no. 11, pp. 2099-2104, 2013.

[16] X. Han and Q. Du, "Research on face recognition based on deep learning," 6th Int. Conf. Digit. Information, Networking, Wirel. Commun. DINWC 2018, pp. 53-58, 2018.

[17] M. Sewak, S. K. Sahay, and H. Rathore, "Comparison of deep learning and the classical machine learning algorithm for the malware detection," Proc. - 2018 IEEE/ACIS 19th Int. Conf. Softw. Eng. Artif. Intell. Netw. Parallel/Distributed Comput. SNPD 2018, pp. 293-296, 2018.

[18] D. R. Monisha and R. Pavithra, "Discriminative Robust Local Binary Pattern Based Face Recognition From A Single Sample Per Person," vol. 16, no. 3, pp. 57-62, 2016.

[19] G. Shakhnarovich, P. A. Viola, and B. Moghaddam, “A unified learning framework for real time face detection and classification," Proc. - 5th IEEE Int. Conf. Autom. Face Gesture Recognition, FGR 2002, pp. 16-23, 2002.

[20] R. W. McGugin and I. Gauthier, Face recognition. 2013.

[21] J. Chang-yeon, "JoFaceDetectionUsingLBPfeatures.pdf,” pp. 1-4, 2008.

[22] R. P. Singh, "Review of Face Detection and Recognition Techniques," Int. J. Comput. Sci. Inf. Technol. Vol. 5 , 2014, 4111-4117 Rev., vol. 3, no. 4, pp. 1008-1013, 2015.

[23] M.-H. Yang, D. J. Kriegman, and N. Ahuja, "Detecting Faces In Image : A Survey - Presentation," IEEE Trans. Pattern Anal. Mach. Intell., vol. 24, no. 1, pp. 1-25, 2002.

[24] L. Fissore, A. Kaltenmeier, P. Laface, G. Micca, and R. Pieraccini, "The Recognition Algorithms," $A d v$. Algorithms Archit. Speech Underst., pp. 7-78, 2011.

[25] A. Roy and S. Marcel, "Haar Local Binary Pattern Feature for Fast Illumination Invariant Face Detection," p. 19.1-19.12, 2012. 